\title{
SYMMETRIC COALITIONAL BINOMIAL SEMIVALUES *
}

\author{
Francesc Carreras ${ }^{\dagger}$ and María Albina Puente ${ }^{\ddagger}$
}

February 2, 2011

\begin{abstract}
We introduce here a family of mixed coalitional values. They extend the binomial semivalues to games endowed with a coalition structure, satisfy the property of symmetry in the quotient game and the quotient game property, generalize the symmetric coalitional Banzhaf value introduced by Alonso and Fiestras and link and merge the Shapley value and the binomial semivalues. A computational procedure in terms of the multilinear extension of the original game is also provided and an application to political science is sketched.
\end{abstract}

Keywords: cooperative game, binomial semivalue, coalition structure, multilinear extension.

Math. Subj. Class. (2000): 91A12.

\section{Introduction}

The parallel axiomatic characterization stated by Feltkamp [28] shows that the only difference between the Shapley value (Shapley [50]) and the Banzhaf value (Owen [40]), as allocation rules for all cooperative games, is that the former satisfies efficiency whereas the latter satisfies the total power property. Differences of this kind become important when one is facing a group decision and negotiation problem and wishes to choose a suitable allocation rule for solving the problem. The properties of a value should always be a main argument for either selecting it or rejecting it in each particular case.

In the framework of cooperative games with a coalition structure, other essential differences also arise between the Owen value (Owen [41]) and the modified Banzhaf value or Owen-Banzhaf value (Owen [43]). The Owen-Banzhaf value fails to satisfy the property of symmetry in the quotient game and the quotient game property, which are satisfied by the Owen value.

\footnotetext{
${ }^{*}$ Research supported by Grant SGR 2009-01029 of the Catalonia Government (Generalitat de Catalunya) and Grants MTM-2006-06064 and MTM 2009-08037 of the Science and Innovation Spanish Ministry and the European Regional Development Fund.

${ }^{\dagger}$ Department of Applied Mathematics II and Industrial and Aeronautical Engineering School of Terrassa, Technical University of Catalonia, Spain. e-mail: francesc.carreras@upc.edu. Corresponding author.

\$Department of Applied Mathematics III and Engineering School of Manresa, Technical University of Catalonia, Spain.e-mail: m.albina.puente@upc.edu
} 
Alonso and Fiestras [4] suggested a modification of the Owen-Banzhaf value that satisfies these two properties and can therefore be compared with the Owen value in terms analogous to Feltkamp [28]. Our aim here is to introduce the notion of coalitional binomial semivalue as a wide generalization of the Alonso-Fiestras value (essentially: $p \in[0,1]$ instead of $p=1 / 2$ ) in order to get the family of symmetric coalitional binomial semivalues that still satisfy the property of symmetry in the quotient game and the quotient game property, so that they differ from the Owen value just in satisfying a total power property instead of efficiency. These new values are especially suited for the study of cooperative games where the players show some (common) tendency as to the size of the coalitions they would agree to form. This tendency is defined by parameter $p$. Let us include a motivating example.

Example 1.1 In Section 5 we apply this family of values to the analysis of an interesting political problem: the Catalonia Parliament during Legislature 2003-2007, prematurely finished in 2006. Curiously, the analysis remains still valid for Legislature 2006-2010 since, in spite of the modification of the seat distribution issued from the elections held in November 1, 2006, the strategic possibilities are exactly the same.

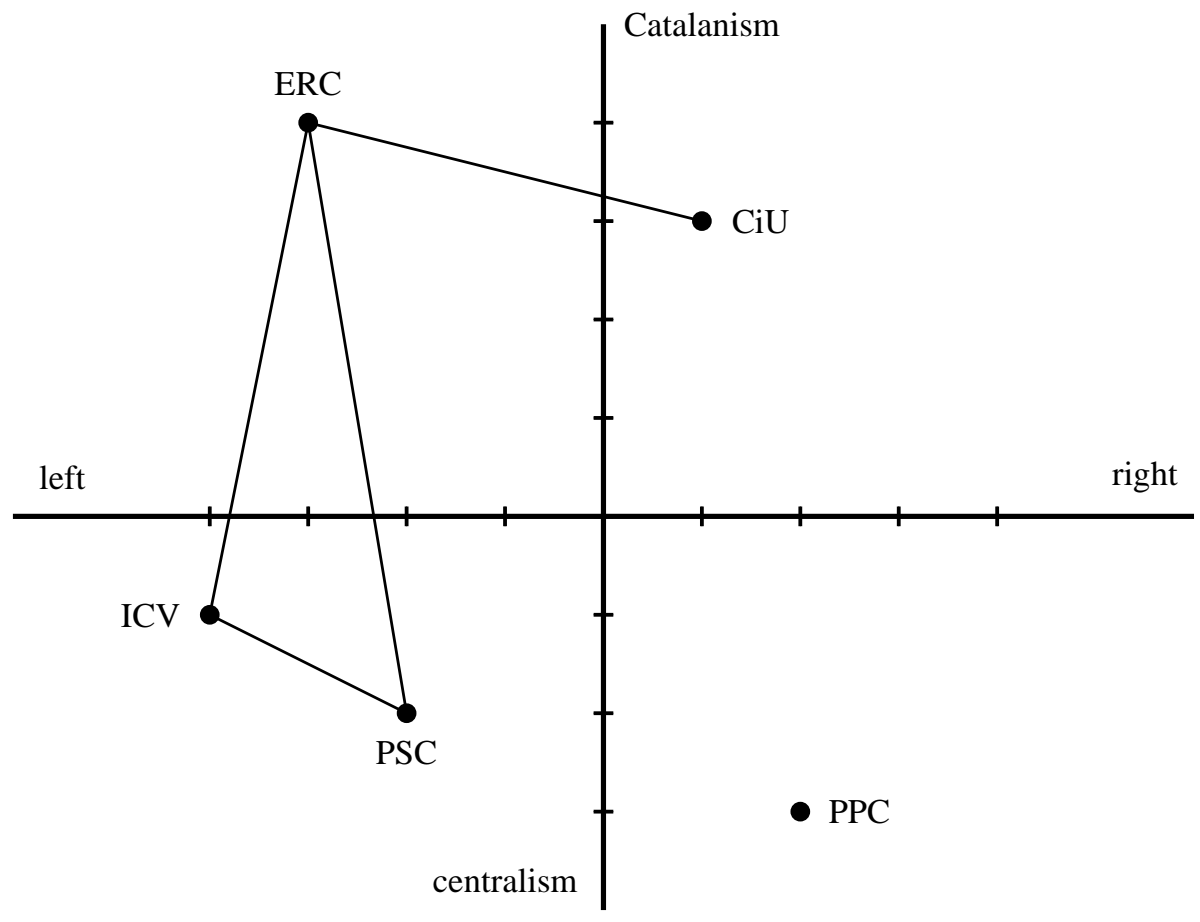

Fig. 1. Political positions in the Catalonia Parliament 2003-2007

In Catalonia, politics is based on two main axes: the classical left-to-right axis and a crossed one going from Spanish centralism to Catalanism (Catalan nationalism) (see Fig. 1). Thus, in 2003 Esquerra Republicana de Catalunya (ERC), a radical nationalist and left-wing 
party, was faced to the dilemma of choosing among either a Catalanist majority coalition with Convergència i Unió (CiU) or a left-wing majority coalition with the Partit dels Socialistes de Catalunya (PSC) and Iniciativa per Catalunya-Verds (ICV), which was finally formed in 2003 and has been repeated in 2006.

A classical approach to study the problem would consist in using either (a) the Shapley value and the Owen value or, alternatively, (b) the Banzhaf value and the Owen-Banzhaf value, in order to evaluate the strategic possibilities of each party in both setups. The results are given in Table 1, where (NO) means no coalition formation, $(\mathrm{C})$ means that $\mathrm{CiU}+\mathrm{ERC}$ forms, and (LW) means that PSC + ERC + ICV forms.

Table 1. Classical measures of power in the Catalonia Parliament 2003-2007

\begin{tabular}{|l|ccc|ccc|}
\hline & \multicolumn{3}{|c|}{$\begin{array}{c}\text { (a) } \\
\text { (b) }\end{array}$} \\
& (NO) & (C) & (LW) & (NO) & (C) & (LW) \\
\hline CiU & 0.4000 & 0.5000 & 0.0000 & 0.6250 & 0.5000 & 0.0000 \\
PSC & 0.2333 & 0.0000 & 0.3889 & 0.3750 & 0.0000 & 0.3750 \\
ERC & 0.2333 & 0.5000 & 0.3889 & 0.3750 & 0.5000 & 0.3750 \\
PPC & 0.0667 & 0.0000 & 0.0000 & 0.1250 & 0.0000 & 0.0000 \\
ICV & 0.0667 & 0.0000 & 0.2222 & 0.1250 & 0.0000 & 0.1250 \\
\hline
\end{tabular}

According to the Shapley and Owen values used in (a), ERC would strictly prefer joining $\mathrm{CiU}$ instead of PSC and ICV. The same conclusion is obtained according to the Banzhaf and Owen-Banzhaf values used in (b). In both cases, the results fail to provide a mathematical explanation of ERC's actual decision (to join PSC and ICV).

Instead, by using binomial semivalues, and symmetric coalitional binomial semivalues whenever a coalition structure exists, the conclusion of the theoretical analysis is that ERC, the crucial agent in this scenario, was not necessarily forced to participate in the left-wing tripartite government but would have got more political power in joining CiU depending on the tendency of the parties. The reader is referred to Example 5.3 for a detailed analysis.

The organization of the paper is as follows. In Section 2, a minimum of preliminaries is provided. Section 3 is devoted to define and study the symmetric coalitional binomial semivalue, and it includes an axiomatic characterization that parallels Owen [41] for the Owen value. In Section 4 we present a computation procedure for the symmetric coalitional binomial semivalue. Section 5 contains a remark on simple games and two detailed examples and, finally, we have included in Section 6 a historical note.

\section{Preliminaries}

\subsection{Games and semivalues}

Let $N$ be a finite set of players and $2^{N}$ be the set of its coalitions (subsets of $N$ ). A cooperative game on $N$ is a function $v: 2^{N} \rightarrow \mathbb{R}$, that assigns a real number $v(S)$ to each coalition $S \subseteq N$, with $v(\emptyset)=0$. A game $v$ is monotonic if $v(S) \leq v(T)$ whenever $S \subseteq T \subseteq N$. A player $i \in N$ is a dummy in $v$ if $v(S \cup\{i\})=v(S)+v(\{i\})$ for all $S \subseteq N \backslash\{i\}$, and null in $v$ if, moreover, 
$v(\{i\})=0$. Two players $i, j \in N$ are symmetric in $v$ if $v(S \cup\{i\})=v(S \cup\{j\})$ for all $S \subseteq$ $N \backslash\{i, j\}$.

Endowed with the natural operations for real-valued functions, i.e. $v+v^{\prime}$ and $\lambda v$ for all $\lambda \in \mathbb{R}$, the set of all cooperative games on $N$ is a vector space $\mathcal{G}_{N}$. For every nonempty coalition $T \subseteq N$, the unanimity game $u_{T}$ is defined by $u_{T}(S)=1$ if $T \subseteq S$ and $u_{T}(S)=0$ otherwise, and it is easily checked that the set of all unanimity games is a basis for $\mathcal{G}_{N}$. Finally, every permutation $\theta$ of $N$ induces a linear automorphism of $\mathcal{G}_{N}$ given by $(\theta v)(S)=$ $v\left(\theta^{-1} S\right)$ for all $S \subseteq N$ and all $v$.

By a value on $\mathcal{G}_{N}$ we will mean a map $f: \mathcal{G}_{N} \rightarrow \mathbb{R}^{N}$, that assigns to every game $v$ a vector $f[v]$ with components $f_{i}[v]$ for all $i \in N$.

Following Weber's [58] axiomatic description, $\psi: \mathcal{G}_{N} \rightarrow \mathbb{R}^{N}$ is a semivalue iff it satisfies the following properties:

(i) linearity: $\psi\left[v+v^{\prime}\right]=\psi[v]+\psi\left[v^{\prime}\right]$ (additivity) and $\psi[\lambda v]=\lambda \psi[v]$ for all $v, v^{\prime} \in \mathcal{G}_{N}$ and $\lambda \in \mathbb{R}$

(ii) anonymity: $\psi_{\theta i}[\theta v]=\psi_{i}[v]$ for all $\theta$ on $N, i \in N$, and $v \in \mathcal{G}_{N}$;

(iii) positivity: if $v$ is monotonic, then $\psi[v] \geq 0$;

(iv) dummy player property: if $i \in N$ is a dummy in game $v$, then $\psi_{i}[v]=v(\{i\})$.

There is an interesting characterization of semivalues, by means of weighting coefficients, due to Dubey, Neyman and Weber [26]. Set $n=|N|$. Then: (a) for every weighting vector $\left\{p_{k}\right\}_{k=0}^{n-1}$ such that $\sum_{k=0}^{n-1} p_{k}\left(\begin{array}{c}n-1 \\ k\end{array}\right)=1$ and $p_{k} \geq 0$ for all $k$, the expression

$$
\psi_{i}[v]=\sum_{S \subseteq N \backslash\{i\}} p_{S}[v(S \cup\{i\})-v(S)] \quad \text { for all } i \in N \text { and all } v \in \mathcal{G}_{N},
$$

where $s=|S|$, defines a semivalue $\psi$; (b) conversely, every semivalue can be obtained in this way; (c) the correspondence given by $\left\{p_{k}\right\}_{k=0}^{n-1} \mapsto \psi$ is bijective.

Thus, the payoff that a semivalue allocates to every player in any game is a weighted sum of his marginal contributions in the game. If $p_{k}$ is interpreted as the probability that a given player $i$ joins a coalition of size $k$, provided that all the coalitions of a common size have the same probability of being joined, then $\psi_{i}[v]$ is the expected marginal contribution of that player to a random coalition he joins.

Well known examples of semivalues are the Shapley value $\varphi$ (Shapley [50]), for which $p_{k}=1 / n\left(\begin{array}{c}n-1 \\ k\end{array}\right)$, and the Banzhaf value $\beta$ (Owen [40]), for which $p_{k}=2^{1-n}$. The Shapley value $\varphi$ is the only efficient semivalue, in the sense that $\sum_{i \in N} \varphi_{i}[v]=v(N)$ for every $v \in \mathcal{G}_{N}$.

Notice that these values are defined for each $N$. The same happens with the binomial semivalues, introduced by Puente [48] as follows. Let $p \in[0,1]$ and $p_{k}=p^{k}(1-p)^{n-k-1}$ for $k=0,1, \ldots, n-1$. Then $\left\{p_{k}\right\}_{k=0}^{n-1}$ is a weighting vector and defines a semivalue that will be denoted as $\psi^{p}$ and called the $p$-binomial semivalue. Using the convention that $0^{0}=1$, the definition makes sense also for $p=0$ and $p=1$, where we respectively get the dictatorial index $\psi^{0}$ and the marginal index $\psi^{1}$, introduced by Owen [42] and such that $\psi_{i}^{0}[v]=v(\{i\})$ and $\psi_{i}^{1}[v]=v(N)-v(N \backslash\{i\})$ for all $i \in N$ and all $v \in \mathcal{G}_{N}$. Of course, $p=1 / 2$ gives $\psi^{1 / 2}=\beta$ - the Banzhaf value.

In fact, semivalues are defined on cardinalities rather than on specific player sets: this means that a weighting vector $\left\{p_{k}\right\}_{k=0}^{n-1}$ defines a semivalue $\psi$ on all $N$ such that $n=|N|$. 
When necessary, we shall write $\psi^{(n)}$ for a semivalue on cardinality $n$ and $p_{k}^{(n)}$ for its weighting coefficients. A semivalue $\psi^{(n)}$ induces semivalues $\psi^{(t)}$ for all cardinalities $t<n$, recurrently defined by the Pascal triangle (inverse) formula given by Dragan [23]:

$$
p_{k}^{(t)}=p_{k}^{(t+1)}+p_{k+1}^{(t+1)} \text { for } 0 \leq k<t .
$$

A series $\psi=\left\{\psi^{(n)}\right\}_{n=1}^{\infty}$ of semivalues, one for each cardinality, is a multisemivalue if it satisfies Dragan's recurrence formula. Thus, the Shapley and Banzhaf values and all binomial semivalues are multisemivalues.

\subsection{Games with coalition structure}

Let us consider a finite set, say, $N=\{1,2, \ldots, n\}$. We will denote by $P(N)$ the set of all partitions of $N$. Each $P \in P(N)$ is called a coalition structure or system of unions on $N$. The so-called trivial coalition structures are $P^{n}=\{\{1\},\{2\}, \ldots,\{n\}\}$ (individual coalitions) and $P^{N}=\{N\}$ (grand coalition). A cooperative game with a coalition structure is a pair $[v ; P]$, where $v \in \mathcal{G}_{N}$ and $P \in P(N)$ for a given $N$. Each partition $P$ gives a pattern of cooperation among players. We denote by $\mathcal{G}_{N}^{c s}$ the set of all cooperative games with a coalition structure and player set $N$.

If $[v ; P] \in \mathcal{G}_{N}^{c s}$ and $P=\left\{P_{1}, P_{2}, \ldots, P_{m}\right\}$, the quotient game $v^{P}$ is the cooperative game played by the unions, or, rather, by the set $M=\{1,2, \ldots, m\}$ of their representatives, as follows:

$$
v^{P}(R)=v\left(\bigcup_{r \in R} P_{r}\right) \quad \text { for all } R \subseteq M .
$$

Unions $P_{r}, P_{s}$ are said to be symmetric in $[v ; P]$ if $r, s$ are symmetric players in $v^{P}$.

By a coalitional value on $\mathcal{G}_{N}^{c s}$ we will mean a map $g: \mathcal{G}_{N}^{c s} \rightarrow \mathbb{R}^{N}$, which assigns to every pair $[v ; P]$ a vector $g[v ; P]$ with components $g_{i}[v ; P]$ for each $i \in N$.

The Owen value (Owen [41]) is the coalitional value $\Phi$ defined by

$$
\Phi_{i}[v ; P]=\sum_{R \subseteq M \backslash\{k\}} \sum_{T \subseteq P_{k} \backslash\{i\}} \frac{1}{m p_{k}} \frac{1}{\left(\begin{array}{c}
m-1 \\
r
\end{array}\right)} \frac{1}{\left(\begin{array}{c}
p_{k}-1 \\
t
\end{array}\right)}[v(Q \cup T \cup\{i\})-v(Q \cup T)]
$$

for all $i \in N$ and $[v ; P] \in \mathcal{G}_{N}^{c s}$, where $P_{k} \in P$ is the union such that $i \in P_{k}$ and $Q=\bigcup_{r \in R} P_{r}$. It was axiomatically characterized by Owen [41] as the only coalitional value that satisfies the following properties: the natural extensions to this framework of

- efficiency

- additivity

- the dummy player property

and also

- symmetry within unions: if $i, j \in P_{k}$ are symmetric in $v$ then

$$
\Phi_{i}[v ; P]=\Phi_{j}[v ; P]
$$


- symmetry in the quotient game: if $P_{r}, P_{s} \in P$ are symmetric in $[v ; P]$ then

$$
\sum_{i \in P_{r}} \Phi_{i}[v ; P]=\sum_{j \in P_{s}} \Phi_{j}[v ; P] .
$$

The Owen value is a coalitional value of the Shapley value $\varphi$ in the sense that $\Phi\left[v ; P^{n}\right]=$ $\varphi[v]$ for all $v \in \mathcal{G}_{N}$. Besides, $\Phi\left[v ; P^{N}\right]=\varphi[v]$. Finally, as $\Phi$ is defined for any $N$, the following property makes sense and is also satisfied:

- quotient game property: for all $[v ; P] \in \mathcal{G}_{N}^{c s}$,

$$
\sum_{i \in P_{k}} \Phi_{i}[v ; P]=\Phi_{k}\left[v^{P} ; P^{m}\right] \quad \text { for all } P_{k} \in P .
$$

The Owen value can be viewed as a two-step allocation rule. First, each union $P_{k}$ receives its payoff in the quotient game according to the Shapley value; then, each $P_{k}$ splits this amount among its players by applying the Shapley value to a game played in $P_{k}$ as follows: the worth of each subcoalition $T$ of $P_{k}$ is the Shapley value that $T$ would get in a "pseudoquotient game" played by $T$ and the remaining unions on the assumption that $P_{k} \backslash T$ leaves the game, i.e. the quotient game after replacing $P_{k}$ with $T$. This is the way to bargain within the union: each subcoalition $T$ claims the payoff it would obtain when dealing with the other unions in absence of its partners in $P_{k}$.

The Owen-Banzhaf value $B$ (Owen [43]) follows a similar scheme. The resulting formula parallels that of the Owen value with the sole change of coefficient $1 / m p_{k}\left(\begin{array}{c}m-1 \\ r\end{array}\right)\left(\begin{array}{c}p_{k}-1 \\ t\end{array}\right)$ by $2^{1-m} 2^{1-p_{k}}$. This value, which is a coalitional value of the Banzhaf value $\beta$, does not satisfy efficiency, but neither symmetry in the quotient game nor the quotient game property. The bargaining interpretation is the same as in the case of the Owen value by replacing everywhere the Shapley value with the Banzhaf value.

Alonso and Fiestras [4] introduced a modification of the Owen-Banzhaf value. In this case, the coefficient of each marginal contribution is replaced with $2^{1-m} / p_{k}\left(p_{k}-1\right)$. This symmetric coalitional Banzhaf value $\Pi$ satisfies the same properties as the Owen value, with the sole exception of efficiency — replaced by a total power property—, as well as the quotient game property, and it is a coalitional value of the Banzhaf value.

Example 2.1 (Alonso and Fiestras [4]) Let us take $n=5$ and consider the unanimity game $u_{N}$ and the coalition structure $P=\left\{P_{1}, P_{2}\right\}$ where $P_{1}=\{1,2,3\}$ and $P_{2}=\{4,5\}$. Notice that the quotient game is $u_{N}^{P}=u_{M}$, where $M=\{1,2\}$. It is not difficult to obtain the following values:

$$
\begin{aligned}
\beta\left[u_{N}\right] & =(1 / 16,1 / 16,1 / 16,1 / 16,1 / 16), \\
\beta\left[u_{N}^{P}\right] & =(1 / 2,1 / 2), \\
B\left[u_{N} ; P\right] & =(1 / 8,1 / 8,1 / 8,1 / 4,1 / 4) .
\end{aligned}
$$

As $P_{1}$ and $P_{2}$ are symmetric in $\left[u_{N} ; P\right]$, it follows that the Owen-Banzhaf value $B$ fails to satisfy the property of symmetry in the quotient game. Neither the quotient game property is fulfilled by $B$ in this instance. Instead

$$
\Pi\left[u_{N} ; P\right]=(1 / 6,1 / 6,1 / 6,1 / 4,1 / 4)
$$


so that both properties are satisfied by the Alonso-Fiestras value $\Pi$ (here and elsewhere).

Since the Banzhaf value $\beta$ is a particular $p$-binomial semivalue $(p=1 / 2)$, this example also shows that the coalitional p-binomial semivalues, which can be obtained from the work by Albizuri and Zarzuelo [3] or Amer and Giménez [8] by applying Owen's scheme to any $p$-binomial semivalue, satisfy, in general, none of both properties. That's why we will generalize Alonso and Fiestras' procedure.

\section{The symmetric coalitional binomial semivalue}

In this section we define and study a "coalitional version" of each $p$-binomial semivalue for games with coalition structure. This includes, besides the explicit formula, an axiomatic characterization and an interpretation in terms of a two-step bargaining process, among unions, first, and among players within each union later. We begin by dealing with binomial semivalues. We recall that $\psi^{p}$ denotes, for each $p \in[0,1]$, the $p$-binomial semivalue acting on a fixed $\mathcal{G}_{N}$.

Definition 3.1 Let $p \in[0,1]$. A value $f$ on $\mathcal{G}_{N}$ satisfies the $p$-binomial total power property if

$$
\sum_{i \in N} f_{i}[v]=\sum_{i \in N} \sum_{S \subseteq N \backslash\{i\}} p^{s}(1-p)^{n-s-1}[v(S \cup\{i\})-v(S)] \text { for all } v \in \mathcal{G}_{N} .
$$

Lemma 3.2 Let $\emptyset \neq S \subseteq N, s=|S|$ and $i \in N$. Then $\psi_{i}^{p}\left[u_{S}\right]=p^{s-1}$ if $i \in S$, and $\psi_{i}^{p}\left[u_{S}\right]=0$ otherwise.

Proof: Let $i \in S$. By the definition of the weighting coefficients of $\psi^{p}$ we have

$$
\begin{aligned}
& \psi_{i}^{p}\left[u_{S}\right]=\left(\begin{array}{c}
n-s \\
0
\end{array}\right) p^{s-1}(1-p)^{n-s}+\left(\begin{array}{c}
n-s \\
1
\end{array}\right) p^{s}(1-p)^{n-s-1}+\cdots+\left(\begin{array}{c}
n-s \\
n-s
\end{array}\right) p^{n-1}= \\
& =p^{s-1}\left[(p+(1-p)]^{n-s}=p^{s-1} .\right.
\end{aligned}
$$

If $i \notin S$, the dummy player property yields $\psi_{i}^{p}\left[u_{S}\right]=0$.

Proposition 3.3 The unique semivalue on $\mathcal{G}_{N}$ that satisfies the p-binomial total power property is the p-binomial semivalue $\psi^{p}$.

In other words, $\sum_{i \in N} \psi_{i}[v]=\sum_{i \in N} \psi_{i}^{p}[v]$ for all $v \in \mathcal{G}_{N}$ implies $\psi=\psi^{p}$.

Proof: (a) It is obvious that the $p$-binomial semivalue $\psi^{p}$ satisfies the $p$-binomial total power property.

(b) Using linearity and the fact that the unanimity games form a basis of $\mathcal{G}_{N}$, it suffices to prove that any semivalue $\psi$ satisfying the $p$-binomial total power property coincides with $\psi^{p}$ on each unanimity game. Let $u_{S}$ be such a game for some $S \subseteq N$. From the dummy player property we get $\psi_{i}\left[u_{S}\right]=0=\psi_{i}^{p}\left[u_{S}\right]$ for all $i \notin S$. From the $p$-binomial total power property it follows that $\sum_{i \in S} \psi_{i}\left[u_{S}\right]=s p^{s-1}$. Using anonymity, $\psi_{i}\left[u_{S}\right]=p^{s-1}$ for each $i \in S$, and this allocation coincides with $\psi_{i}^{p}\left[u_{S}\right]$ according to Lemma 3.2. 
In particular, setting $p=1 / 2$ we obtain that the Banzhaf value $\beta$ is the only semivalue that satisfies the classical total power property:

$$
\sum_{i \in N} f_{i}[v]=\frac{1}{2^{n-1}} \sum_{i \in N} \sum_{S \subseteq N \backslash\{i\}}[v(S \cup\{i\})-v(S)] \quad \text { for all } v \in \mathcal{G}_{N} .
$$

The Owen (resp., Owen-Banzhaf) value is a natural extension of the Shapley (resp., Banzhaf) value to games with a coalition structure. We generalize this idea.

Definition 3.4 Given a value $f$ on $\mathcal{G}_{N}$, a coalitional value of $f$ is a coalitional value $g$ on $\mathcal{G}_{N}^{c s}$ such that $g\left[v ; P^{n}\right]=f[v]$ for all $v \in \mathcal{G}_{N}$.

Let $g$ be a coalitional value of the $p$-binomial semivalue $\psi^{p}$ defined for all $N$, and assume that $g$ satisfies the quotient game property. Then, for a given $N$ and any $[v ; P] \in \mathcal{G}_{N}^{c s}$,

$$
\begin{aligned}
\sum_{i \in N} g_{i}[v ; P] & =\sum_{k \in M} \sum_{i \in P_{k}} g_{i}[v ; P]=\sum_{k \in M} g_{k}\left[v^{P} ; P^{m}\right]=\sum_{k \in M} \psi_{k}^{p}\left[v^{P}\right]= \\
& =\sum_{k \in M} \sum_{R \subseteq M \backslash\{k\}} p^{r}(1-p)^{m-r-1}\left[v^{P}(R \cup\{k\})-v^{P}(R)\right] .
\end{aligned}
$$

This motivates the next definition, that is an adaptation of the $p$-binomial total power property to games with a coalition structure.

Definition 3.5 Let $p \in[0,1]$. A coalitional value $g$ on $\mathcal{G}_{N}^{c s}$ satisfies the coalitional p-binomial total power property if, for all $[v ; P] \in \mathcal{G}_{N}^{c s}$,

$$
\sum_{i \in N} g_{i}[v ; P]=\sum_{k \in M} \sum_{R \subseteq M \backslash\{k\}} p^{r}(1-p)^{m-r-1}\left[v^{P}(R \cup\{k\})-v^{P}(R)\right] .
$$

The next statement defines and axiomatically characterizes, for each $p \in[0,1]$, the symmetric coalitional $p-$ binomial semivalue, which will be denoted as $\Omega^{p}$.

Theorem 3.6 Let $p \in[0,1]$. For any $N$ there is a unique coalitional value on $\mathcal{G}_{N}^{c s}$ that satisfies additivity, the dummy player property, symmetry within unions, symmetry in the quotient game, and the coalitional p-binomial total power property. Given $[v ; P] \in \mathcal{G}_{N}^{c s}$, this value allocates to each player $i \in N$ the real number

$$
\Omega_{i}^{p}[v ; P]=\sum_{R \subseteq M \backslash\{k\}} \sum_{T \subseteq P_{k} \backslash\{i\}} p^{r}(1-p)^{m-r-1} \frac{1}{p_{k}\left(\begin{array}{c}
p_{k}-1 \\
t
\end{array}\right)}[v(Q \cup T \cup\{i\})-v(Q \cup T)],
$$

where $P_{k} \in P$ is the union such that $i \in P_{k}$ and $Q=\bigcup_{r \in R} P_{r}$.

Moreover, $\Omega^{p}$ is a coalitional value of the p-binomial semivalue $\psi^{p}$ and satisfies the quotient game property.

Proof: (a) (Existence) It suffices to show that the coalitional value $\Omega^{p}$ given by the above formula satisfies the five properties enumerated in the statement.

1. Additivity. It merely follows from the expression of $\Omega_{i}^{p}[v ; P]$. 
2. Dummy player property. Let $i \in N$ be a dummy player in game $v$ and $P$ be any coalition structure. Assume $i \in P_{k}$. Then $v(Q \cup T \cup\{i\})-v(Q \cup T)=v(\{i\})$ for all $R$ and $T$. As, moreover,

$$
\sum_{R \subseteq M \backslash\{k\}} p^{r}(1-p)^{m-r-1}=1 \quad \text { and } \quad \sum_{T \subseteq P_{k} \backslash\{i\}} \frac{1}{p_{k}\left(\begin{array}{c}
p_{k}-1 \\
t
\end{array}\right)}=1,
$$

we conclude that $\Omega_{i}^{p}[v ; P]=v(\{i\})$.

3. Symmetry within unions. Let $i, j \in P_{k} \in P$ be symmetric players in game $v$. For each $R \subseteq M \backslash\{k\}$ and $T \subseteq P_{k} \backslash\{i, j\}$, let $\Delta(R, T, h)=v(Q \cup T \cup\{h\})-v(Q \cup T)$ for $h=i, j$. Then, by the symmetric position of $i, j$ in $v$,

$$
\begin{aligned}
& f(R, T)=\Delta(R, T, i)-\Delta(R, T, j)=0 \quad \text { and } \\
& g(R, T)=\Delta(R, T \cup\{j\}, i)-\Delta(R, T \cup\{i\}, j)=0,
\end{aligned}
$$

so that

$$
\Omega_{i}^{p}[v ; P]-\Omega_{j}^{p}[v ; P]=\sum_{R \subseteq M \backslash\{k\}} p^{r}(1-p)^{m-r-1} \sum_{T \subseteq P_{k} \backslash\{i, j\}}\left[\frac{f(R, T)}{p_{k}\left(\begin{array}{c}
p_{k}-1 \\
t
\end{array}\right)}+\frac{g(R, T)}{p_{k}\left(\begin{array}{c}
p_{k}-1 \\
t+1
\end{array}\right)}\right]=0 .
$$

4. Coalitional $p$-binomial total power property. Let $[v ; P] \in \mathcal{G}_{N}^{c s}$. Fixing $k \in M$, for every $R \subseteq M \backslash\{k\}$ we consider the game $v_{R} \in \mathcal{G}_{P_{k}}$ defined by

$$
v_{R}(T)=v(Q \cup T)-v(Q) \quad \text { for all } T \subseteq P_{k} .
$$

The Shapley value gives, for each $i \in P_{k}$,

$$
\varphi_{i}\left[v_{R}\right]=\sum_{T \subseteq P_{k} \backslash\{i\}} \frac{1}{p_{k}\left(\begin{array}{c}
p_{k}-1 \\
t
\end{array}\right)}[v(Q \cup T \cup\{i\})-v(Q \cup T)] .
$$

Using the efficiency of $\varphi$, we get

$$
\sum_{i \in P_{k}} \varphi_{i}\left[v_{R}\right]=v_{R}\left(P_{k}\right)=v\left(Q \cup P_{k}\right)-v(Q)=v^{P}(R \cup\{k\})-v^{P}(R) .
$$

Hence

$$
\sum_{i \in P_{k}} \Omega_{i}^{p}[v ; P]=\sum_{R \subseteq M \backslash\{k\}} p^{r}(1-p)^{m-r-1}\left[v^{P}(R \cup\{k\})-v^{P}(R)\right]=\left(\psi^{p}\right)_{k}^{(m)}\left[v^{P}\right]
$$

and, finally,

$$
\sum_{i \in N} \Omega_{i}^{p}[v ; P]=\sum_{k \in M} \sum_{R \subseteq M \backslash\{k\}} p^{r}(1-p)^{m-r-1}\left[v^{P}(R \cup\{k\})-v^{P}(R)\right] .
$$

5. Symmetry in the quotient game. It readily follows from the relationship

$$
\sum_{i \in P_{k}} \Omega_{i}^{p}[v ; P]=\left(\psi^{p}\right)_{k}^{(m)}\left[v^{P}\right],
$$


stated in the previous point, and the anonymity (whence symmetry) of the $p$-binomial semivalue $\psi^{p}$.

(b) (Uniqueness) Let $g$ be a coalitional value on $\mathcal{G}_{N}^{c s}$ satisfying the five properties. Using additivity and the fact that the unanimity games form a basis of $\mathcal{G}_{N}$, it suffices to show that $g$ is completely determined by its action on any pair of the form $\left[\lambda u_{S} ; P\right]$, where $\lambda \in \mathbb{R}, \emptyset \neq S \subseteq N$ and $P \in P(N)$.

By the dummy player property, $g_{i}\left[\lambda u_{S} ; P\right]=0$ if $i \notin S$. This leaves us with players $i \in S$.

Let $S^{\prime}=\left\{k \in M: S \cap P_{k} \neq \emptyset\right\}$ and, for every $k \in S^{\prime}, S_{k}^{\prime}=S \cap P_{k}$. It is easy to see that $\left(\lambda u_{S}\right)^{P}=\lambda u_{S^{\prime}}$. From the coalitional $p$-binomial total power property, and applying Lemma 3.2, we have

$$
\sum_{i \in N} g_{i}\left[\lambda u_{S} ; P\right]=\sum_{k \in M}\left(\psi^{p}\right)_{k}^{(m)}\left[\lambda u_{S^{\prime}}\right]=\sum_{k \in S^{\prime}}\left(\psi^{p}\right)_{k}^{(m)}\left[\lambda u_{S^{\prime}}\right]=\lambda s^{\prime} p^{s^{\prime}-1} .
$$

Now, from symmetry in the quotient game, if $k \in S^{\prime}$ then

$$
\sum_{i \in S_{k}^{\prime}} g_{i}\left[\lambda u_{S} ; P\right]=\sum_{i \in P_{k}} g_{i}\left[\lambda u_{S} ; P\right]=\lambda p^{s^{\prime}-1}
$$

and, finally, using symmetry within unions,

$$
g_{i}\left[\lambda u_{S} ; P\right]=\frac{\lambda p^{s^{\prime}-1}}{s_{k}^{\prime}} \quad \text { for any } i \in S_{k}^{\prime} .
$$

As $S=\bigcup_{k \in S^{\prime}} S_{k}^{\prime}$, this concludes the proof that $g$ is univocally determined.

(c) $\Omega^{p}$ is a coalitional value of the $p$-binomial semivalue $\psi^{p}$. Indeed, for $P=P^{n}$ the explicit formula of $\Omega^{p}$ reduces to

$$
\Omega_{i}^{p}\left[v ; P^{n}\right]=\sum_{R \subseteq N \backslash\{i\}} p^{r}(1-p)^{m-r-1}[v(R \cup\{i\})-v(R)]=\psi_{i}^{p}[v] .
$$

Finally, the quotient game property: as we have seen when showing the symmetry in the quotient game in part (a) of this proof, and using the preceding property for $\mathcal{G}_{M}^{c s}$,

$$
\sum_{i \in P_{k}} \Omega_{i}^{p}[v ; P]=\left(\psi^{p}\right)_{k}^{(m)}\left[v^{P}\right]=\Omega_{k}^{p}\left[v^{P} ; P^{m}\right] .
$$

Remark 3.7 (a) The symmetric coalitional $p$-binomial semivalue is a natural (and wide) generalization of Alonso and Fiestras' symmetric coalitional Banzhaf value, since $\Omega^{1 / 2}=\Pi$.

(b) $\Omega^{p}$ relates not only to the $p$-binomial semivalue $\psi^{p}$ (of which it is a coalitional value) but also to the Shapley value $\varphi$, as

$$
\Omega^{p}\left[v ; P^{N}\right]=\varphi[v] \quad \text { for any } v \in \mathcal{G}_{N} .
$$

Thus, in some manner, $\Omega^{p}$ establishes a "coalitional path" that links $\varphi$ and $\psi^{p}$.

(c) From Theorem 3.6 it follows that the only axiomatic difference between the Owen value $\Phi$ and the symmetric coalitional $p$-binomial semivalue $\Omega^{p}$ is that the former satisfies efficiency whereas the latter satisfies the coalitional $p$-binomial total power property, in a 
way that parallels the distinction between the Shapley value $\varphi$ and the $p$-binomial semivalue $\psi^{p}$.

(d) It is worth mentioning that in the parallel axiomatizations of the Owen value and the symmetric coalitional $p$-binomial semivalue, additivity might be replaced with linearity, and the dummy player property with the null player property. We have chosen the first possibility in each case (additivity and dummy player property).

(e) The symmetric coalitional $p$-binomial semivalue also merges the Shapley value and the $p$-binomial semivalue. It is the result of a two-step bargaining procedure similar to that of the Owen value. In our case, the unions play the quotient game among themselves and each one receives the payoff given by the $p$-binomial semivalue $\psi^{p}$, and then this payoff is efficiently shared within the union according to the Shapley value $\varphi$.

\section{A computation procedure}

The multilinear extension (Owen [39]) of a game $v \in \mathcal{G}_{N}$ is the real-valued function defined on $\mathbb{R}^{N}$ by

$$
f\left(x_{1}, x_{2}, \ldots, x_{n}\right)=\sum_{S \subseteq N} \prod_{i \in S} x_{i} \prod_{j \notin S}\left(1-x_{j}\right) v(S) .
$$

As is well known, both the Shapley and Banzhaf values of any game $v$ can be easily obtained from its multilinear extension. Indeed, $\varphi[v]$ can be calculated by integrating the partial derivatives of the multilinear extension of the game along the main diagonal $x_{1}=$ $x_{2}=\cdots=x_{n}$ of the cube $[0,1]^{N}$ (Owen [39]), while the partial derivatives of that multilinear extension evaluated at point $(1 / 2,1 / 2, \ldots, 1 / 2)$ give $\beta[v]$ (Owen [40]. This latter procedure extends well to any $p$-binomial semivalue (see Puente [48], Freixas and Puente [29] or Amer and Giménez [8]) by evaluating the derivatives at point $(p, p, \ldots, p)$.

In the context of games with a coalition structure, the multilinear extension technique has been also applied to computing the Owen value $\Phi$ (Owen and Winter [45]), as well as the Owen-Banzhaf value $B$ (Carreras and Magaña [18]) and the symmetric coalitional Banzhaf value $\Pi$ (Alonso, Carreras and Fiestras [5]). In this section we present a method to compute the symmetric coalitional $p$-binomial semivalue $\Omega^{p}$ by means of the multilinear extension of the game.

Theorem 4.1 Let $p \in[0,1]$ and $[v ; P] \in \mathcal{G}_{N}^{c s}$ be a cooperative game with a coalition structure. Then the following steps lead to the symmetric coalitional p-binomial semivalue of any player $i \in P_{k}$ in $[v ; P]$.

1. Obtain the multilinear extension $f\left(x_{1}, x_{2}, \ldots, x_{n}\right)$ of game $v$.

2. For every $r \neq k$ and all $h \in P_{r}$, replace the variable $x_{h}$ with $y_{r}$. This yields a new function of $x_{j}$ for $j \in P_{k}$ and $y_{r}$ for $r \in M \backslash\{k\}$.

3. In this function, reduce to 1 all higher exponents, i.e. replace with $y_{r}$ each $y_{r}^{q}$ such that $q>1$. This gives a new multilinear function that we denote as $g\left(\left(x_{j}\right)_{j \in P_{k}},\left(y_{r}\right)_{r \in M \backslash\{k\}}\right)$. 
4. In the function obtained in step 3, substitute each $y_{r}$ by $p$. This provides a new function $\alpha_{k}\left(\left(x_{j}\right)_{j \in P_{k}}\right)$ defined by

$$
\alpha_{k}\left(\left(x_{j}\right)_{j \in P_{k}}\right)=g\left(\left(x_{j}\right)_{j \in P_{k}},(p)_{r \in M \backslash\{k\}}\right) .
$$

5. Finally, the symmetric coalitional p-binomial semivalue of player $i \in P_{k}$ in $[v ; P]$ is given by

$$
\Omega_{i}^{p}[v ; P]=\int_{0}^{1} \frac{\partial \alpha_{k}}{\partial x_{i}}(z, z, \ldots, z) d z .
$$

Proof: Steps 1-3 have already been used by Owen and Winter [45], Carreras and Magaña [18] and Alonso, Carreras and Fiestras [5] in dealing with the Owen value, the Owen-Banzhaf value and the symmetric coalitional Banzhaf value, respectively. It will be useful to recall their common argument here.

By the second and third steps, we get a multilinear function where all terms corresponding to coalitions $S$ such that $S \cap P_{r} \neq \emptyset$ and $(N \backslash S) \cap P_{r} \neq \emptyset$ for some $r \in M \backslash\{k\}$ vanish. Indeed, in step 2, the terms corresponding to these coalitions include expressions like $c y_{r}^{q_{1}}\left(1-y_{r}\right)^{q_{2}}$, with $q_{1}, q_{2} \in \mathbb{N}$, and in step 3 these terms turn on $c\left(y_{r}-y_{r}\right)$ thus getting zero.

Hence, the only coalitions $S$ for which the corresponding term of the (initial) multilinear extension may not vanish after steps 2 and 3 are those of the form $S=Q \cup T$, where $T \subseteq P_{k}$ and $Q=\bigcup_{r \in R} P_{r}$ for some $R \subseteq M \backslash\{k\}$. The function arising from step 3 is therefore

$$
\begin{gathered}
g\left(\left(x_{j}\right)_{j \in P_{k}},\left(y_{r}\right)_{r \in M \backslash\{k\}}\right)= \\
\sum_{T \subseteq P_{k}} \sum_{R \subseteq M \backslash\{k\}} \prod_{j \in T} x_{j} \prod_{j \in P_{k} \backslash T}\left(1-x_{j}\right) \prod_{r \in R} y_{r} \prod_{r \notin R \cup\{k\}}\left(1-y_{r}\right) v(Q \cup T) .
\end{gathered}
$$

Substituting each $y_{r}$ by $p$ (step 4) gives

$$
\alpha_{k}\left(\left(x_{j}\right)_{j \in P_{k}}\right)=\sum_{T \subseteq P_{k}} \sum_{R \subseteq M \backslash\{k\}} \prod_{j \in T} x_{j} \prod_{j \in P_{k} \backslash T}\left(1-x_{j}\right) p^{r}(1-p)^{m-r-1} v(Q \cup T) .
$$

By differentiating function $\alpha_{k}\left(\left(x_{j}\right)_{j \in P_{k}}\right)$ with respect to $x_{i}$

$$
\begin{gathered}
\frac{\partial \alpha_{k}}{\partial x_{i}}\left(\left(x_{j}\right)_{j \in P_{k}}\right)= \\
\sum_{T \subseteq P_{k} \backslash\{i\}} \sum_{R \subseteq M \backslash\{k\}} \prod_{j \in T} x_{j} \prod_{j \in P_{k} \backslash(T \cup\{i\})}\left(1-x_{j}\right) p^{r}(1-p)^{m-r-1}[v(Q \cup T \cup\{i\})-v(Q \cup T)] .
\end{gathered}
$$

Finally, by step 5 ,

$$
\begin{gathered}
\int_{0}^{1} \frac{\partial \alpha_{k}}{\partial x_{i}}(z, z, \ldots, z) d z= \\
\sum_{T \subseteq P_{k} \backslash\{i\}} \sum_{R \subseteq M \backslash\{k\}} p^{r}(1-p)^{m-r-1}[v(Q \cup T \cup\{i\})-v(Q \cup T)] \int_{0}^{1} z^{t}(1-z)^{p_{k}-t-1} d z= \\
\sum_{T \subseteq P_{k} \backslash\{i\}} \sum_{R \subseteq M \backslash\{k\}} p^{r}(1-p)^{m-r-1} \frac{t !\left(p_{k}-t-1\right) !}{p_{k} !}[v(Q \cup T \cup\{i\})-v(Q \cup T)]=\Omega_{i}^{p}[v ; P] .
\end{gathered}
$$




\section{A remark and two examples}

Simple games form an especially interesting class of cooperative games. Not only as a test bed for many cooperative concepts, but also for the variety of their interpretations, often far from game theory. In particular, they are frequently applied to describe and analyze collective decision-making mechanisms - weighted majority games play a crucial role here-, and the notion of voting power is closely attached to them.

Shapley and Shubik [52] were the first to adapt a cooperative tool — the Shapley valueto simple games, using it as a measure of power. Shapley [51] stated a series of arguments inviting to a self-contained treatment of this class of games, and Dubey [24] initiated this line when providing an axiomatic characterization of the Shapley-Shubik power index as a solution concept on the class of simple games, for which he introduced the transfer property in order to replace the useless additivity property.

A cooperative game $v$ on $N$ is simple if it is monotonic and $v(S)=0$ or 1 for every $S \subseteq N$. A coalition $S \subseteq N$ is winning in $v$ if $v(S)=1$ (otherwise it is called losing), and $W(v)$ denotes the set of winning coalitions in $v$. Due to monotonicity, the set $W^{m}(v)$ of all minimal winning coalitions determines $W(v)$ and hence the game. A simple game $v$ is a weighted majority game if there are nonnegative weights $w_{1}, w_{2}, \ldots, w_{n}$ allocated to the players and a positive quota $q$ such that

$$
v(S)=1 \quad \text { iff } \quad \sum_{i \in S} w_{i} \geq q .
$$

We then write $v=\left[q ; w_{1}, w_{2}, \ldots, w_{n}\right]$. (For additional details on simple games, we refer the interested reader to $e$.g. Carreras and Freixas [14], Taylor and Zwicker [54] or Carreras [12].)

Let $\mathcal{S} \mathcal{G}_{N}$ denote the set of all simple games on a given player set $N$. A power index on $\mathcal{S} \mathcal{G}_{N}$ is a function $f: S \mathcal{G}_{N} \rightarrow \mathbb{R}^{N}$. All properties stated for values in this paper -with the sole exception of additivity and linearity - make sense for power indices. As $S \mathcal{G}_{N}$ is a lattice under the standard composition laws given by $\left(v \vee v^{\prime}\right)(S)=\max \left\{v(S), v^{\prime}(S)\right\}$ and $\left(v \wedge v^{\prime}\right)(S)=\min \left\{v(S), v^{\prime}(S)\right\}$, we will say that a power index $f$ satisfies the transfer property if

$$
f\left[v \vee v^{\prime}\right]=f[v]+f\left[v^{\prime}\right]-f\left[v \wedge v^{\prime}\right] \quad \text { for all } v, v^{\prime} \in \mathcal{S} \mathcal{G}_{N} .
$$

Carreras, Freixas and Puente [20] gave an axiomatic characterization and a combinatorial description in terms of weighting coefficients for (the restrictions of) semivalues as power indices, which parallel the corresponding ones for semivalues on general cooperative games.

Let $S \mathcal{G}_{N}^{c s}$ be the set of all simple games with a coalition structure on $N$. A coalitional power index on $\mathcal{S} \mathcal{G}_{N}^{c s}$ is a function $g: \mathcal{S} \mathcal{G}_{N}^{c s} \rightarrow \mathbb{R}^{N}$. All properties stated for coalitional values in this paper - excluding again additivity and linearity-, as well as the natural extension of the transfer property, make sense for coalitional power indices. Vázquez, van den Nouweland and García-Jurado [56] carried out an axiomatic characterization of the (restricted) Owen value as a coalitional power index by means of efficiency, the transfer property, the dummy player property, symmetry within unions and symmetry in the quotient game.

In a similar way, we have found a "parallel" axiomatic characterization of the symmetric coalitional binomial semivalues as power indices (that is, restricted to $\mathcal{S} \mathcal{G}_{N}^{c S}$ ) that we state without proof because it is very similar to that of Theorem 3.6. 
Corollary 5.1 Let $p \in[0,1]$. For any $N$ there is a unique coalitional power index on $\mathcal{S} \mathcal{G}_{N}^{c s}$ that satisfies the coalitional p-binomial total power property, the transfer property, the dummy player property, symmetry within unions and symmetry in the quotient game. It is the restriction of the symmetric coalitional p-binomial semivalue $\Omega^{P}$ to $\mathcal{S} \mathcal{G}_{N}^{c s}$.

Besides, this index satisfies the quotient game property and reduces to the (restricted) $p-$ binomial semivalue $\psi^{p}$ when $P=P^{n}$ and to the Shapley-Shubik power index $\varphi$ when $P=P^{N}$.

Example 5.2 Let us consider the 5-person weighted majority game

$$
v=[68 ; 46,42,23,15,9]
$$

and the coalition structure $P=\{\{1,4\},\{2,5\},\{3\}\}$. We will compute $\psi^{p}[v]$ and $\Omega^{p}[v ; P]$ for any $p \in[0,1]$.

The set of minimal winning coalitions of the game is

$$
W^{m}(v)=\{\{1,2\},\{1,3\},\{1,4,5\},\{2,3,4\},\{2,3,5\}\}
$$

and the multilinear extension of $v$ is

$$
\begin{aligned}
& f\left(x_{1}, x_{2}, x_{3}, x_{4}, x_{5}\right)=x_{1} x_{2}+x_{1} x_{3}-x_{1} x_{2} x_{3}+x_{1} x_{4} x_{5}+x_{2} x_{3} x_{4}+x_{2} x_{3} x_{5}- \\
& -x_{1} x_{2} x_{3} x_{4}-x_{1} x_{2} x_{3} x_{5}-x_{1} x_{2} x_{4} x_{5}-x_{1} x_{3} x_{4} x_{5}-x_{2} x_{3} x_{4} x_{5}+2 x_{1} x_{2} x_{3} x_{4} x_{5} .
\end{aligned}
$$

Taking into account that players 2 and 3, on one hand, and players 4 and 5 on the other, are symmetric in $v$, the computation method for binomial semivalues stated by Puente [48] (see also Amer and Giménez [8]) gives

$$
\begin{aligned}
& \psi_{1}^{p}[v]=\frac{\partial f}{\partial x_{1}}(p, p, p, p, p)=p(1-p)\left(2+2 p-2 p^{2}\right), \\
& \psi_{3}^{p}[v]=\psi_{2}^{p}[v]=\frac{\partial f}{\partial x_{2}}(p, p, p, p, p)=p(1-p)\left(1+2 p-2 p^{2}\right), \\
& \psi_{5}^{p}[v]=\psi_{4}^{p}[v]=\frac{\partial f}{\partial x_{4}}(p, p, p, p, p)=p(1-p)\left(2 p-2 p^{2}\right) .
\end{aligned}
$$

In order to compute $\Omega^{p}[v]$ we use Theorem 4.1. As $M=\{1,2,3\}$, steps $1-3$ give

$$
\begin{aligned}
g_{1}\left(x_{1}, x_{4}, y_{2}, y_{3}\right) & =y_{2} y_{3}+x_{1}\left(y_{2}+y_{3}-2 y_{2} y_{3}\right), \\
g_{2}\left(y_{1}, x_{2}, x_{5}, y_{3}\right) & =y_{1} y_{3}+x_{2}\left(y_{1}-y_{1} y_{3}\right)+x_{5}\left(y_{1}-y_{1} y_{3}\right)+x_{2} x_{5}\left(y_{3}-y_{1}\right), \\
g_{3}\left(y_{1}, y_{2}, x_{3}\right) & =y_{1} y_{2}+x_{3}\left(y_{1}+y_{2}-2 y_{1} y_{2}\right),
\end{aligned}
$$

and step 4 leads to

$$
\begin{aligned}
\alpha_{1}\left(x_{1}, x_{4}\right) & =g_{1}\left(x_{1}, x_{4}, p, p\right)=p^{2}+x_{1}\left(2 p-2 p^{2}\right), \\
\alpha_{2}\left(x_{2}, x_{5}\right) & =g_{2}\left(p, x_{2}, x_{5}, p\right)=p^{2}+x_{2}\left(p-p^{2}\right)+x_{5}\left(p-p^{2}\right), \\
\alpha_{3}\left(x_{3}\right) & =g_{3}\left(p, p, x_{3}\right)=p^{2}+x_{3}\left(2 p-2 p^{2}\right) .
\end{aligned}
$$


Step 5 concludes the procedure and gives

$$
\begin{aligned}
& \Omega_{1}^{p}[v ; P]=\int_{0}^{1} \frac{\partial \alpha_{1}}{\partial x_{1}}(z, z) d z=2 p-2 p^{2}, \quad \Omega_{4}^{p}[v ; P]=\int_{0}^{1} \frac{\partial \alpha_{1}}{\partial x_{4}}(z, z) d z=0 \\
& \Omega_{2}^{p}[v ; P]=\int_{0}^{1} \frac{\partial \alpha_{2}}{\partial x_{2}}(z, z) d z=p-p^{2}, \quad \Omega_{5}^{p}[v ; P]=\int_{0}^{1} \frac{\partial \alpha_{2}}{\partial x_{5}}(z, z) d z=p-p^{2} \\
& \Omega_{3}^{p}[v ; P]=\int_{0}^{1} \frac{\partial \alpha_{3}}{\partial x_{3}}(z) d z=2 p-2 p^{2} .
\end{aligned}
$$

Thus

$$
\Omega^{p}[v ; P]=\left(2 p-2 p^{2}, p-p^{2}, 2 p-2 p^{2}, 0, p-p^{2}\right) .
$$

In the following example, we shall apply some values and coalitional values (mainly $\psi^{p}$ and $\Omega^{p}$ ) to the analysis of an interesting political structure: the Catalonia Parliament 20032007. All values have been computed using the multilinear extension technique, as illustrated in the preceding example.

In the papers by Straffin [53], Laruelle [34] and Laruelle and Valenciano [35], the Banzhaf value $\beta$ is suggested as a power measure more suitable than the Shapley value. The natural generalization to semivalues has been argued by Laruelle and Valenciano [36], Carreras and Freixas [17], and Carreras, Freixas and Puente [20]. By considering here binomial semivalues, we look at the Banzhaf value in perspective, as will be shown by the results of our analysis.

Therefore, our study of alliances will be based on the bargaining process corresponding to the symmetric coalitional binomial semivalues $\Omega^{p}$ : first, a power notion is shared among unions in the quotient game by means of the Banzhaf value or a binomial semivalue; then, the power so got by each union is shared among its members by using the Shapley value. This will reflect that both bargaining steps are of different nature. Indeed, notice that, once an alliance is formed - and, especially, if it supports a coalition government-, cabinet ministries, parliamentary and institutional positions, budgets, and other political responsibilities have to be distributed efficiently among the parties of the coalition, hence in a way as closely as possible to the one suggested by the Shapley value. At this point, the quotient game property and symmetry in the quotient game become very relevant properties. In fact, they are connected because if a coalitional value satisfies the quotient game property (as is the case for all $\Omega^{p}$ ) and it is a coalitional value of the Banzhaf value (or a $p$-binomial semivalue) then symmetry in the quotient game follows from the anonymity of $\beta$ (or of $\psi^{p}$ ).

Which is the reason for letting $p$ range from 0 to 1 ? Notice that a reasonable regularity assumption on players' behavior is that the probability to form coalitions follows a monotonic (increasing or decreasing) behavior. Then, it is not difficult to see that the only semivalues such that $p_{k+1}=\lambda p_{k}$ for all $k$ (maybe the simplest form of monotonicity) are precisely the $p$-binomial semivalues, in which case $\lambda=\frac{p}{1-p}$ for any $p \in[0,1]$. For example, $p=0.1$ means that the players are very reticent to form coalitions, whereas $p=0.8$ means that great coalitions are likelier. The neutral case $p=0.5$ corresponds to the Banzhaf value. Table 2 shows, for $n=5$, the weighting coefficients of $\psi^{p}$ for several values of $p$. 
Table 2. Weighting coefficients of some $p$-binomial semivalues $\psi^{p}$ for $n=5$

\begin{tabular}{|l|l|l|c|l|}
\hline & $p=0.1$ & $p=0.4$ & $\begin{array}{c}p=0.5 \\
\text { (Banzhaf) }\end{array}$ & $p=0.8$ \\
\hline$p_{0}=(1-p)^{4}$ & 0.6561 & 0.1296 & 0.0625 & 0.0016 \\
\hline$p_{1}=p(1-p)^{3}$ & 0.0729 & 0.0864 & 0.0625 & 0.0064 \\
\hline$p_{2}=p^{2}(1-p)^{2}$ & 0.0081 & 0.0576 & 0.0625 & 0.0256 \\
\hline$p_{3}=p^{3}(1-p)$ & 0.0009 & 0.0384 & 0.0625 & 0.1024 \\
\hline$p_{4}=p^{4}$ & 0.0001 & 0.0256 & 0.0625 & 0.4096 \\
\hline
\end{tabular}

As we will see, almost all allocations $\psi_{i}^{p}[v]$ and coalitional allocations $\Omega_{i}^{p}[v ; P]$ will show factors $p(1-p)$; the sole exceptions are the cases where $i$ is a dictator or veto player (if we were dealing with improper games, we should add winner players to this short list). Furthermore, the maximum or the minimum of all these allocations for each player will be attained in case $p=0.5$, that respectively correspond to the Banzhaf value $\beta=\psi^{1 / 2}$ or to the AlonsoFiestras coalitional value $\Pi=\Omega^{1 / 2}$. These properties would not have been discovered if only the case $p=0.5$ were considered.

Example 5.3 (The Catalonia Parliament, Legislature 2003-2007) Five parties elected members to the Catalonia Parliament (135 seats) in the elections held on November 16, 2003, giving rise to a seat distribution that can be represented by the weighted majority game

$$
v=[68 ; 46,42,23,15,9]
$$

Let us briefly describe ideologically the agents in this game:

1: CiU (Convergència i Unió), Catalan nationalist middle-of-the-road coalition of two federated parties.

2: PSC (Partit dels Socialistes de Catalunya), moderate left-wing socialist party, federated to the Partido Socialista Obrero Español.

3: ERC (Esquerra Republicana de Catalunya), radical Catalan nationalist left-wing party.

4: PPC (Partit Popular de Catalunya), conservative party, Catalan delegation of the Partido Popular.

5: ICV (Iniciativa per Catalunya-Verds), coalition of Catalan eurocommunist parties, federated to Izquierda Unida, and ecologist groups ("Verds").

Notice that, as pointed out in Example 5.2,

$$
W^{m}(v)=\{\{1,2\},\{1,3\},\{1,4,5\},\{2,3,4\},\{2,3,5\}\},
$$

so that players 2 and 3 on one hand, and 4 and 5 on the other, are symmetric in $v$.

We show in Table 3 the evaluation of $v$ given by several binomial semivalues $\psi^{p}$. The total power is $\tau^{p}[v]=\sum_{i \in N} \psi_{i}^{p}[v]$. 
Table 3. Initial power distribution in the Catalonia Parliament 2003-2007

\begin{tabular}{llcccc} 
& $\psi_{i}^{p}[v]$ & $p=0.1$ & $p=0.4$ & $p=0.5$ & $p=0.8$ \\
\hline 1. CiU & $p(1-p)\left(2+2 p-2 p^{2}\right)$ & 0.1962 & 0.5952 & 0.6250 & 0.3712 \\
2. PSC & $p(1-p)\left(1+2 p-2 p^{2}\right)$ & 0.1062 & 0.3552 & 0.3750 & 0.2112 \\
3. ERC & $p(1-p)\left(1+2 p-2 p^{2}\right)$ & 0.1062 & 0.3552 & 0.3750 & 0.2112 \\
4. PPC & $p(1-p)\left(2 p-2 p^{2}\right)$ & 0.0162 & 0.1152 & 0.1250 & 0.0512 \\
5. ICV & $p(1-p)\left(2 p-2 p^{2}\right)$ & 0.0162 & 0.1152 & 0.1250 & 0.0512 \\
\hline$\tau^{p}[v]$ & $p(1-p)\left(4+10 p-10 p^{2}\right)$ & 0.4410 & 1.5360 & 1.6250 & 0.8960
\end{tabular}

It is easy to see that the allocations found for $p$ and $1-p$ would coincide because the game is decisive (proper and strong). Notice that the proportions between the allocations to the players decrease as $p$ approaches 0.5 from any of the extreme possibilities ( 0 or 1$)$. Also notice that the maximum allocation (power) for any player and the maximum total power are got for $p=0.5$ (Banzhaf value).

Next we are interested in the study and comparison of several coalition structures. In each case, we have computed the coalitional value $\Omega^{p}$ for all $p \in[0,1]$ and also $\Pi$ (for $p=1 / 2$ ) and the coalitional $p$-binomial total power $T^{p}[v ; P]=\sum_{i \in N} \Omega_{i}^{p}[v ; P]$. The cases and results are as follows:

- The left-wing alliance PSC+ICV, as a previous step. The corresponding coalition structure is $P=\{\{2,5\},\{1\},\{3\},\{4\}\}$, and the coalitional values are:

$$
\begin{aligned}
\Pi[v ; P] & =(1 / 2,3 / 8,1 / 2,0,1 / 8), \\
\Omega^{p}[v ; P] & =\left(2 p-2 p^{2}, 1.5 p-1.5 p^{2}, 2 p-2 p^{2}, 0,0.5 p-0.5 p^{2}\right), \\
T^{p}[v ; P] & =6 p(1-p) .
\end{aligned}
$$

- The simultaneous alliances $\mathrm{CiU}+\mathrm{PPC}$ and PSC+ICV, as an alternative previous step. The corresponding coalition structure is $P=\{\{1,4\},\{2,5\},\{3\}\}$, and the coalitional values are:

$$
\begin{aligned}
\Pi[v ; P] & =(1 / 2,1 / 4,1 / 2,0,1 / 4), \\
\Omega^{p}[v ; P] & =\left(2 p-2 p^{2}, p-p^{2}, 2 p-2 p^{2}, 0, p-p^{2}\right), \\
T^{p}[v ; P] & =6 p(1-p) .
\end{aligned}
$$

- The left-wing majority alliance PSC+ERC+ICV. The corresponding coalition structure is $P=\{\{2,3,5\},\{1\},\{4\}\}$, and the coalitional values are:

$$
\begin{aligned}
\Pi[v ; P] & =(0,5 / 12,5 / 12,0,2 / 12), \\
\Omega^{p}[v ; P] & =\left(0, \frac{1+p-p^{2}}{3}, \frac{1+p-p^{2}}{3}, 0, \frac{1-2 p+2 p^{2}}{3}\right), \\
T^{p}[v ; P] & =1 .
\end{aligned}
$$

Notice that $\Omega_{i}^{p}[v ; P]>\psi_{i}^{p}[v]$ for all $p \in[0,1]$ and $i=2,3,5$, and also that $p=0.5$ gives the maximum of $\Omega^{p}[v ; P]$ for PSC and ERC but, at the same time, the minimum of $\Omega^{p}[v ; P]$ for ICV. 
Incidentally, in this case $B[v ; P]=(0,3 / 8,3 / 8,0,1 / 8)$, so that $B$ fails to satisfy the quotient game property and the sharing of the dictatorial power is by no means convincing because of its inefficiency.

- The catalanist majority alliance CiU+ERC. The corresponding coalition structure is $P=\{\{1,3\},\{2\},\{4\},\{5\}\}$, and the coalitional values are:

$$
\begin{aligned}
\Pi[v ; P] & =(5 / 8,0,3 / 8,0,0), \\
\Omega^{p}[v ; P] & =\left(\frac{1+p-p^{2}}{2}, 0, \frac{1-p+p^{2}}{2}, 0,0\right), \\
T^{p}[v ; P] & =1 .
\end{aligned}
$$

In this case $\Pi_{i}[v ; P]=\beta_{i}[v]$ but $\Omega_{i}^{p}[v ; P]>\psi_{i}^{p}[v]$ for all $p \in[0,1]$ and $i=1,3$ (unless $p=0.5$, where the equality holds). Here $p=0.5$ gives the maximum of $\Omega^{p}[v ; P]$ for $\mathrm{CiU}$ and the minimum for ERC.

A most convenient way to analyze this set of evaluations of the coalitional behavior will consist in considering different values of $p$, and we will take $0.1,0.4,0.5$ (this gives $\Pi$ ) and 0.8. Tables 4-7 show all these particular allocations but we prefer the following order: $p=0.5, p=0.4, p=0.8$ and $p=0.1$.

Table 4. Evaluation according to $\psi^{p}$ and $\Omega^{p}$ for $p=0.5$

\begin{tabular}{llccccc} 
scenario & value & $\mathrm{CiU}$ & $\mathrm{PSC}$ & $\mathrm{ERC}$ & $\mathrm{PPC}$ & $\mathrm{ICV}$ \\
\hline initial (no alliance) & $\beta$ & 0.6250 & 0.3750 & 0.3750 & 0.1250 & 0.1250 \\
PSC+ICV & $B=\Pi$ & 0.5000 & 0.3750 & 0.5000 & 0 & 0.1250 \\
PSC+ICV and CiU+PPC & $B=\Pi$ & 0.5000 & 0.2500 & 0.5000 & 0 & 0.2500 \\
PSC+ERC+ICV & $B$ & 0 & 0.3750 & 0.3750 & 0 & 0.1250 \\
PSC+ERC+ICV & $\Pi$ & 0 & 0.4167 & 0.4167 & 0 & 0.1667 \\
CiU+ERC & $B=\Pi$ & 0.6250 & 0 & 0.3750 & 0 & 0
\end{tabular}

In Table 4, we find that precoalition PSC+ICV does not increase the power of their members, but it damages the strategic position of $\mathrm{CiU}$ and enhances the strategic possibilities of ERC. The alternative (simultaneous precoalitions PSC+ICV and CiU+PPC) does not make better off CiU and PPC but, instead, damages PSC, increases the power of ICV and gives the same position to ERC. Of course, PPC loses its small power even in joining CiU (its only natural partner in this situation) because once PSC+ICV is formed PPC becomes a null player.

An important point arises when considering the majority formation. According to the Owen-Banzhaf value $B$, forming a winning coalition does not change the power of its members with regard to the initial distribution, although it serves to reduce the outside parties to a null position. Instead, from the viewpoint of the symmetric coalitional Banzhaf value $\Pi$, coalition PSC+ERC+ICV clearly increases the power of each one of its members, and hence it suggests to ERC the convenience to choose this coalition (which also satisfies its partners, PSC and ICV) instead of CiU+ERC. 
Therefore, we have to point out here that after a short period of negotiations, precisely concerning these two options, alliance PSC+ERC+ICV was actually formed and got the regional government of Catalonia, ending 23 years of $\mathrm{CiU}$ governments headed by Jordi Pujol (under absolute majority of this party or with the parliamentary support of PPC). The actual sharing of positions gave the presidency of the government to Pasqual Maragall (PSC) but the presidency of the Parliament and the "Conseller en cap" position (a sort of vice-presidency of the government) to Ernest Benach and Josep Lluis Carod Rovira (both ERC), respectively. The remaining cabinet positions ("conselleries") were distributed in the proportion 8:5:2 among the three parties.

Table 5. Evaluation according to $\psi^{p}$ and $\Omega^{p}$ for $p=0.4$

\begin{tabular}{lccccc} 
scenario & $\mathrm{CiU}$ & PSC & ERC & PPC & ICV \\
\hline initial (no alliance) & 0.5952 & 0.3552 & 0.3552 & 0.1152 & 0.1152 \\
PSC+ICV & 0.4800 & 0.3600 & 0.4800 & 0 & 0.1200 \\
PSC+ICV and CiU+PPC & 0.4800 & 0.2400 & 0.4800 & 0 & 0.2400 \\
PSC+ERC+ICV & 0 & 0.4133 & 0.4133 & 0 & 0.1733 \\
CiU+ERC & 0.6200 & 0 & 0.3800 & 0 & 0
\end{tabular}

We recall that the allocations on this (decisive) game for a given $p$ are the same as for $1-p$, so that our comments on Table $5(p=0.4)$ are the same as they would be for $p=0.6$, and the analogue holds for Tables 6 and 7.

By comparing the results given in Table 4 with those of Table 5, where it is assumed that players are not indifferent to join a coalition of any size but, rather, they prefer not too big coalitions (as $p=0.4$ ), we notice that forming precoalition PSC+ICV increases a bit the power of its members. Its effects on the outside parties, as well as those of the alternative (simultaneous formation of $\mathrm{CiU}+\mathrm{PPC}$ ) are the same as in Table 4.

As to the formation of majorities, here, not only in the case of PSC+ERC+ICV but also in the case of CiU+ERC, every party entering such a coalition clearly increases its power. However, from ERC's viewpoint, coalition PSC+ERC+ICV gives again the best fraction of coalitional power.

Table 6. Evaluation according to $\psi^{p}$ and $\Omega^{p}$ for $p=0.8$

\begin{tabular}{lccccc} 
scenario & CiU & PSC & ERC & PPC & ICV \\
\hline initial (no alliance) & 0.3712 & 0.2112 & 0.2112 & 0.0512 & 0.0512 \\
PSC+ICV & 0.3200 & 0.2400 & 0.3200 & 0 & 0.0800 \\
PSC+ICV and CiU+PPC & 0.3200 & 0.1600 & 0.3200 & 0 & 0.1600 \\
PSC+ERC+ICV & 0 & 0.3867 & 0.3867 & 0 & 0.2267 \\
CiU+ERC & 0.5800 & 0 & 0.4200 & 0 & 0
\end{tabular}

It is worth mentioning that almost all (initial or coalitional) power allocations given in Table 6 are lower than in the previous cases. The only exceptions are for ICV in PSC+ERC+ICV and ERC in CiU+ERC. Nevertheless, the variations undergone by the initial allocations when the precoalitions form are similar to those found in Table 5. The new feature here 
is that, in these circumstances $(p=0.8)$, ERC would clearly prefer CiU+ERC instead of $\mathrm{PSC}+\mathrm{ERC}+\mathrm{ICV}$.

Table 7. Evaluation according to $\psi^{p}$ and $\Omega^{p}$ for $p=0.1$

\begin{tabular}{lccccc} 
scenario & CiU & PSC & ERC & PPC & ICV \\
\hline initial (no alliance) & 0.1962 & 0.1062 & 0.1062 & 0.0162 & 0.0162 \\
PSC+ICV & 0.1800 & 0.1350 & 0.1800 & 0 & 0.0450 \\
PSC+ICV and CiU+PPC & 0.1800 & 0.0900 & 0.1800 & 0 & 0.0900 \\
PSC+ERC+ICV & 0 & 0.3633 & 0.3633 & 0 & 0.2733 \\
CiU+ERC & 0.5450 & 0 & 0.4550 & 0 & 0
\end{tabular}

Finally, Table 7 exhibits the same trends as Table 6 but they are even strengthened. Again, ERC would prefer CiU+ERC, and notice that the increase of its power in agreeing to form this coalition would be greater than in the previous case.

It is not difficult to see that ERC would prefer option PSC+ERC+ICV instead of CiU+ERC if, and only if, $p \in\left(\frac{5-\sqrt{5}}{10}, \frac{5+\sqrt{5}}{10}\right)$, would remain indifferent if $p=\frac{5 \pm \sqrt{5}}{10}$ and would prefer CiU+ERC if $p \notin\left[\frac{5-\sqrt{5}}{10}, \frac{5+\sqrt{5}}{10}\right]$.

As a conclusion of our analysis, we find that the evaluation of games and games with a coalition structure by means of binomial semivalues and symmetric coalitional binomial semivalues provides a new approach to the study of the coalitional bargaining. Some general properties sketched only on the basis of this instance should deserve further attention. And, finally, the extension of the coalitional theory to probabilistic values might be, in the near future, an interesting research field.

\section{A historical note}

Shapley [50] (see also Roth [49] and Owen [44]) initiated the value theory for cooperative games. The Shapley value applies without restrictions and provides, for every game, a single payoff vector to the players. The restriction of the value to simple games gives rise to the Shapley-Shubik [52] power index, that was axiomatized by Dubey [24] introducing the transfer property. As a sort of reaction, Banzhaf [11] proposed a different power index (see also Coleman [21], and even Penrose [47]), that Owen [40] extended to a dummy-independent and somehow "normalized" Banzhaf value for all cooperative games. A nice almost common characterization of the Shapley and Banzhaf values would be given by Feltkamp [28], and a sound interpretative and comparative analysis has been carried out by Laruelle and Valenciano [35]. See also Owen [42], Dubey and Shapley [25], Lehrer [38], Dragan [22] and Carreras [13].

Dubey, Neyman and Weber [26] axiomatically introduced the notion of semivalue, that encompasses both the Shapley and Banzhaf values (see also Weber [57] and Einy [27]). Weber [58] gave an alternative characterization for semivalues and introduced the probabilistic values by dropping anonymity.

Many authors have been working on semivalues. We will refer only to the most related to the present paper. Carreras and Freixas [15] and [16] introduced regular semivalues. 
Puente [48] devoted much of her Ph.D. thesis to semivalues and introduced binomial semivalues as a natural generalization of the Banzhaf value (see also Freixas and Puente [29], where different ways are provided to evaluate the importance of the components in a given reliability system based on semivalues and probabilistic values). Laruelle and Valenciano [36] and Carreras, Freixas and Puente [20] investigated semivalues as power indices, that is, by restricting them to simple games. Finally, Carreras and Freixas [17] suggested several applications of semivalues based on their versatility.

Games with a coalition structure were introduced by Aumann and Drèze [10], who extended the Shapley value to this new framework in such a manner that the game really splits into subgames played by the unions isolatedly from each other, and every player receives the payoff allocated by the restriction of the Shapley value to the subgame he is playing within his union. A second approach was used by Owen [41], when introducing and axiomatically characterizing his coalitional value (Owen value). In this case, the unions play a quotient game among themselves, and each one receives a payoff which, in turn, is shared among its players in an internal game. Both payoffs, in the quotient game for unions and within each union for its players, are given by applying the Shapley value. Further axiomatizations of the Owen value have been given by e.g. Hart and Kurz [33], Peleg [46], Winter [59], Amer and Carreras [6] and [7], Vázquez, van den Nouweland and García-Jurado [56], Vázquez [55], Hamiache [31] and [32], Albizuri and Zarzuelo [3] and Albizuri [2].

By applying a similar procedure to the Banzhaf value, Owen [43] got the modified Banzhaf value or Owen-Banzhaf value for games with a coalition structure. In this case the payoffs at both levels, that of the unions in the quotient game and that of the players within each union, are given by the Banzhaf value. This modified value was axiomatically characterized only recently, by Albizuri [1] and [2] and, independently, by Amer, Carreras and Giménez [9]. Interesting interpretations of this value as a power index when restricted to simple games can be found in Laruelle and Valenciano [37].

The natural generalization of semivalues for games with coalition structure has been carried out by Albizuri and Zarzuelo [3]. These authors provide axiomatic characterizations in both cases: the homogeneous one, when a common semivalue is used by unions in the quotient game and by players within each one of them (see also Giménez [30] and Amer and Giménez [8] for this case), and the heterogeneous one, where different semivalues apply in the quotient game and (uniformly) within all unions.

Alonso and Fiestras [4] realized that the Owen-Banzhaf value fails to satisfy two interesting properties of the Owen value: symmetry in the quotient game and the quotient game property. Then they suggested to modify the two-step allocation scheme and use the Banzhaf value for sharing in the quotient game and the Shapley value within unions. This gave rise to a new, heterogeneous coalitional value that can be compared with the Owen value and satisfies the above properties.

In fact, heterogeneous coalitional values are a particular case of mixed coalitional values. Mixed coalitional values were already suggested by Carreras and Magaña [19] in a more general setup (see also Alonso, Carreras and Fiestras [5]). The idea is that unions might use any value in the quotient game and, then, the players of each union might use a value different from that of the unions and from those used within other unions. Notice that the unions are, in general, of a different nature from the original, single players, and even from each other, and the quotient game may well possess features not found in the initial game. 
The question is not, therefore, "why will the unions follow, as entities, a way different from players' one?" but, rather, "why not?" After all, freedom is a human aspiration that we should take into account in our mathematical modeling of the real life behavior, and the contract for forming each union could (in fact, it should) perfectly specify the way to share profits among its members.

Then, a formal notation will help us to better distinguish the several coalitional evaluation criteria that can arise. Let $\sigma$ be the semivalue used by unions and $\rho_{1}, \rho_{2}, \ldots, \rho_{m}$ be the semivalues respectively used by each union. We denote the compound rule as

$$
\sigma \rho_{1}, \rho_{2}, \ldots, \rho_{m}
$$

With this notation, a first level of homogeneity is achieved in case $\sigma \rho, \rho, \ldots, \rho=\sigma \rho^{m}$ for some common $\rho$. Thus, Alonso and Fiestras' [4] symmetric coalitional Banzhaf value is $\Pi=\beta \varphi^{m}$, whereas the symmetric coalitional binomial semivalue introduced in this paper is $\Omega^{p}=\psi^{p} \varphi^{m}$, where $\psi^{p}$ is the binomial semivalue defined by number $p$. A further homogeneity level is finally found in the case where $\sigma=\rho$, like in Owen's classical extensions: the Owen value is $\Phi=\varphi \varphi^{m}$ and the Owen-Banzhaf value is $B=\beta \beta^{m}$.

Great attention has also been paid to the computation of values, usually in terms of the multilinear extension (Owen [39]) of the original game. Thus, Owen [39] refers to the Shapley value $\varphi$, Owen [40] to the Banzhaf value $\beta$, and Owen and Winter [45] to the Owen value $\Phi$. Carreras and Magaña [18] have applied the same procedure to the Owen-Banzhaf value $B$, and Carreras and Magaña [19] have studied the multilinear extension of the quotient game.

More recently, Alonso, Carreras and Fiestras [5] apply the multilinear extension method to the Alonso-Fiestras value $\Pi$, and also to a "counterpart" value $M=\varphi \beta^{m}$ introduced by Amer, Carreras and Giménez [9]. In Puente [48] it is shown that the binomial semivalues can be computed in a way very close to that of the Banzhaf value. Giménez [30] and Amer and Giménez [8] prove that (a) any other semivalue requires using a geometrical reference system of the semivalue simplex, given by any $n$ different binomial semivalues, and a linear map whose matrix depends on (the partial derivatives of the multilinear extension of) the game and the reference system - it also applies, of course, to the Shapley value, with no integration step-, and (b) the homogeneous coalitional semivalues can be computed by means of a bilinear form whose matrix depends, again, on the game and the reference system. It is worthy mentioning here that in the case of coalitional binomial semivalues Carreras and Magaña's [18] procedure applies as well.

\section{Acknowledgment}

The authors wish to thank an anonymous referee for his/her positive evaluation and helpful comments.

\section{References}

[1] Albizuri, M.J. [2001]: "An axiomatization of the modified Banzhaf-Coleman index." International Journal of Game Theory 30, 167-176. 
[2] Albizuri, M.J. [2002]: "Axiomatizations of Owen value without efficiency.” Discussion Paper 25, Department of Applied Economics IV, Basque Country University, Spain.

[3] Albizuri, M.J. and Zarzuelo, J.M. [2004]: “On coalitional semivalues.” Games and Economic Behavior 49, 221-243.

[4] Alonso, J.M. and Fiestras, M.G. [2002]: "Modification of the Banzhaf value for games with a coalition structure." Annals of Operations Research 109, 213-227.

[5] Alonso, J.M., Carreras, F. and Fiestras, M.G. [2005]: "The multilinear extension and the symmetric coalition Banzhaf value." Theory and Decision 59, 111-126.

[6] Amer, R. and Carreras, F. [1995]: “Cooperation indices and coalition value.” TOP 3, $117-135$.

[7] Amer, R. and Carreras, F. [2001]: "Power, cooperation indices and coalition structures." In: Power Indices and Coalition Formation (M.J. Holler and G. Owen, eds.), Kluwer, 153-173.

[8] Amer, R. and Giménez, J.M. [2003]: "Modification of semivalues for games with coalition structures." Theory and Decision 54, 185-205.

[9] Amer, R., Carreras, F. and Giménez, J.M. [2002]: "The modified Banzhaf value for games with a coalition structure: an axiomatic characterization." Mathematical Social Sciences 43, 45-54.

[10] Aumann, R.J. and Drèze, J. [1974]: "Cooperative games with coalition structures." International Journal of Game Theory 3, 217-237.

[11] Banzhaf, J.F. [1965]: "Weigthed voting doesn't work: A mathematical analysis." Rutgers Law Review 19, 317-343.

[12] Carreras, F. [2001]: "Elementary theory of simple games." Working Paper MA2-IT01-00001, Department of Applied Mathematics II, Technical University of Catalonia, Spain.

[13] Carreras, F. [2005]: “A decisiveness index for simple games." European Journal of Operational Research 163, 370-387.

[14] Carreras, F. and Freixas, J. [1996]: “Complete simple games.” Mathematical Social Sciences 32, 139-155.

[15] Carreras, F. and Freixas, J. [1999]: "Some theoretical reasons for using (regular) semivalues.” In: Logic, Game Theory and Social Choice (H. de Swart, ed.), Tilburg University Press, 140-154.

[16] Carreras, F. and Freixas, J. [2000]: “A note on regular semivalues.” International Game Theory Review 2, 345-352.

[17] Carreras, F. and Freixas, J. [2002]: "Semivalue versatility and applications." Annals of Operations Research 109, 343-358. 
[18] Carreras, F. and Magaña, A. [1994]: "The multilinear extension and the modified Banzhaf-Coleman index." Mathematical Social Sciences 28, 215-222.

[19] Carreras, F. and Magaña, A. [1997]: "The multilinear extension of the quotient game." Games and Economic Behavior 18, 22-31.

[20] Carreras, F., Freixas, J. and Puente, M.A. [2003]: “Semivalues as power indices.” European Journal of Operational Research 149, 676-687.

[21] Coleman, J.S. [1971]: "Control of collectivities and the power of a collectivity to act." In: Social Choice (B. Lieberman, ed.), Gordon and Breach, New York, 269-300.

[22] Dragan, I. [1996]: “New mathematical properties of the Banzhaf value.” European Journal of Operational Research 95, 451-463.

[23] Dragan, I. [1997]: "Some recursive definitions of the Shapley value and other linear values of cooperative TU games.” Working paper 328, University of Texas at Arlington, United States of America.

[24] Dubey, P. [1975]: “On the uniqueness of the Shapley value.” International Journal of Game Theory 4, 131-139.

[25] Dubey, P. and Shapley, L.S. [1979]: "Mathematical properties of the Banzhaf power index." Mathematics of Operations Research 4, 99-131.

[26] Dubey, P., Neyman, A. and Weber, R.J. [1981]: "Value theory without efficiency.” Mathematics of Operations Research 6, 122-128.

[27] Einy, E. [1987]: "Semivalues of simple games.” Mathematics of Operations Research 12, 185-192.

[28] Feltkamp, V. [1995]: "Alternative axiomatic characterizations of the Shapley and Banzhaf values.” International Journal of Game Theory 24, 179-186.

[29] Freixas, J. and Puente, M.A. [2002]: "Reliability importance measures of the components in a system based on semivalues and probabilistic values." Annals of Operations Research 109, 331-342.

[30] Giménez, J.M. [2001]: Contribuciones al estudio de soluciones para juegos cooperativos (in Spanish). Ph.D. Thesis. Technical University of Catalonia, Spain.

[31] Hamiache, G. [1999]: "A new axiomatization of the Owen value for games with coalition structures." Mathematical Social Sciences 37, 281-305.

[32] Hamiache, G. [2001]: “The Owen value values friendship.” International Journal of Game Theory 29, 517-532.

[33] Hart, S. and Kurz, M. [1983]: "Endogeneous formation of coalitions.” Econometrica 51, 1047-1064. 
[34] Laruelle, A. [1999]: "On the choice of a power index." IVIE Discussion Paper WPAD99-10, Instituto Valenciano de Investigaciones Económicas, Valencia, Spain.

[35] Laruelle, A. and Valenciano, F. [2001a]: "Shapley-Shubik and Banzhaf indices revisited." Mathematics of Operations Research 26, 89-104.

[36] Laruelle, A. and Valenciano, F. [2001b]: "Semivalues and voting power." Discussion Paper 13, Department of Applied Economics IV, Basque Country University, Spain.

[37] Laruelle, A. and Valenciano, F. [2003]: "On the meaning of the Owen-Banzhaf coalitional value in voting situations." Discussion Paper 35, Department of Applied Economics IV, Basque Country University, Spain.

[38] Lehrer, E. [1988]: "An axiomatization of the Banzhaf value." International Journal of Game Theory 17, 89-99.

[39] Owen, G. [1972]: “Multilinear extensions of games." Management Science 18, 64-79.

[40] Owen, G. [1975]: "Multilinear extensions and the Banzhaf value." Naval Research Logistics Quarterly 22, 741-750.

[41] Owen, G. [1977]: "Values of games with a priori unions." In: Mathematical Economics and Game Theory (R. Henn and O. Moeschlin, eds.), Springer, 76-88.

[42] Owen, G. [1978]: "Characterization of the Banzhaf-Coleman index." SIAM Journal of Applied Mathematics 35, 315-327.

[43] Owen, G. [1982]: "Modification of the Banzhaf-Coleman index for games with a priori unions." In: Power, Voting and Voting Power (M.J. Holler, ed.), 232-238.

[44] Owen, G. [1995]: Game Theory. Academic Press Inc., 3d. edition.

[45] Owen, G. and Winter, E. [1992]: "Multilinear extensions and the coalitional value." Games and Economic Behavior 4, 582-587.

[46] Peleg, B. [1989]: "Introduction to the theory of cooperative games. Chapter 8: The Shapley value.” RM 88, Center for Research in Mathematical Economics and Game Theory, the Hebrew University, Israel.

[47] Penrose, L.S. [1946]: "The elementary statistics of majority voting." Journal of the Royal Statistical Society 109, 53-57.

[48] Puente, M.A. [2000]: Aportaciones a la representabilidad de juegos simples y al cálculo de soluciones de esta clase de juegos (in Spanish). Ph.D. Thesis. Technical University of Catalonia, Spain.

[49] Roth, A.E. (ed.) [1988]: The Shapley Value: Essays in Honor of Lloyd S. Shapley. Cambridge University Press.

[50] Shapley, L.S. [1953]: "A value for n-person games.” In: Contributions to the Theory of Games II (H.W. Kuhn and A.W. Tucker, eds.), Princeton University Press, 307-317. 
[51] Shapley, L.S. [1962]: "Simple games: An outline of the descriptive theory." Behavioral Science 7, 59-66.

[52] Shapley, L.S. and Shubik, M. [1954]: "A method for evaluating the distribution of power in a committee system." American Political Science Review 48, 787-792.

[53] Straffin, P.D. [1988]: “The Shapley-Shubik and Banzhaf power indices.” In: The Shapley Value: Essays in Honor of Lloyd S. Shapley (A.E. Roth, ed.), Cambridge University Press, 71-81.

[54] Taylor, A.D. and Zwicker, W.S. [1999]: Simple Games: Desirability Relations, Trading, and Pseudoweightings. Princeton University Press.

[55] Vázquez, M. [1998]: Contribuciones a la teoría del valor en juegos con utilidad transferible (in Spanish). Ph.D. Thesis. University of Santiago de Compostela, Spain.

[56] Vázquez, M., Nouweland, A. van den, and García-Jurado, I. [1997]: “Owen's coalitional value and aircraft landing fees." Mathematical Social Sciences 34, 273-286.

[57] Weber, R.J. [1979]: "Subjectivity in the valuation of games." In: Game Theory and Related Topics (O. Moeschlin and D. Pallaschke, eds.), North-Holland, 129-136.

[58] Weber, R.J. [1988]: "Probabilistic values for games.” In: The Shapley Value: Essays in Honor of Lloyd S. Shapley (A.E. Roth, ed.), Cambridge University Press, 101-119.

[59] Winter, E. [1992]: "The consistency and potential for values with coalition structure." Games and Economic Behavior 4, 132-144. 